\title{
Clinical and Laboratory Features of Streptococcus salivarius Meningitis: A Case Report and Literature Review
}

\author{
Megan Wilson, MD; Ryan Martin, MD; Seth T. Walk, PhD; Carol Young, MT(ASCP); \\ Sylvia Grossman, MT(ASCP); Erin Lin McKean, MD; and David M. Aronoff, MD
}

\begin{abstract}
Streptococcus salivarius is a normal member of the human oral microbiome that is an uncommon cause of invasive infections. Meningitis is a rare but increasingly reported infection caused by $S$. salivarius. Despite the growing number of reported cases, a comprehensive review of the literature on S. salivarius meningitis is lacking. We sought to gain a better understanding of the clinical presentation, evaluation, management, and outcome of $S$. salivarius meningitis by analyzing previously reported cases. In addition to a single case reported here, 64 previously published cases of meningitis were identified for this review. The collected data confirm that most patients presented with classical signs and symptoms of bacterial meningitis with a predominance of neutrophils in the cerebrospinal fluid (CSF) and hypoglycorrhachia. The majority of cases followed iatrogenic or traumatic CSF contamination. Most cases were diagnosed by CSF culture within one day of symptom onset. There was no clear evidence of predisposing co-morbid conditions in patients with meningitis, although in most case reports, limited information was given on the medical history of each patient. Outcomes were generally favorable with antibiotic management. Clinicians should suspect $S$. salivarius meningitis in patients presenting acutely after medical or surgical procedures involving the meninges.
\end{abstract}

Keywords: Streptococcus salivarius, Meningitis, Sinusitis, Viridans Streptococci, Cerebrospinal fluid, Hypoglycorrhachia

Corresponding Author:

David M. Aronoff, MD

46I8-C Medical Sciences Building II

1150 West Medical Center Drive

Ann Arbor, MI 48109-5623

Tel: 734-615-3604

Fax: 734-763-4168

Email: daronoff@umich.edu

Received: February 27, 201 I

Revised:April 26, 2011

Accepted: May II, 20I I

doi: $10.3121 / \mathrm{cmr} .2011 .1001$
A cute bacterial meningitis remains an important cause of morbidity and mortality worldwide, despite advances in prophylactic vaccination and pharmacotherapy. While Haemophilus influenzae was previously the most frequent cause of acute bacterial meningitis in the United States, widespread vaccination against this pathogen has resulted in Streptococcus pneumoniae supplanting $H$. influenzae as the leading cause of bacterial meningitis. ${ }^{1}$ Vaccination against $S$. pneumoniae is now reducing the incidence of central nervous system infections caused by this bacterium. ${ }^{2}$

As the burden of pneumococcal meningitis wanes, a relative increase in the proportion of cases caused by other streptococci emerges. An important example is Streptococcus salivarius, a viridans group streptococcus that is prone to causing nosocomial or iatrogenic central nervous system infections. ${ }^{3}$ As recently underscored by the Centers for Disease Control and Prevention, S. salivarius and other viridans group streptococci are the most frequent causes of bacterial meningitis following spinal procedures such as anesthesia, accounting for up to $60 \%$ of cases. $^{4-6}$ Several case reports describe $S$. salivarius meningitis also complicating upper respiratory infections, endocarditis, post-traumatic 


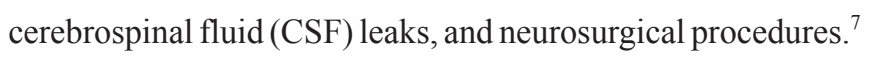
Despite the growing number of reported cases, a comprehensive review of the literature on $S$. salivarius meningitis is lacking.

We recently diagnosed a case of spontaneous $S$. salivarius meningitis associated with a CSF leak that likely resulted from chronic sinus infections. Interestingly, this case was associated with a false-positive urine rapid immunochromatographic test (ICT) for $S$. pneumoniae, although in vitro testing failed to identify an ICT crossreaction between $S$. salivarius and $S$. pneumoniae. We sought to report this interesting clinical occurrence and provide a comprehensive overview of previous cases of $S$. salivarius meningitis that summarize key features of this infection.

\section{Methods}

The $S$. salivarius reference strain ATCC13419 was obtained from the American Tissue Type Collection (Manassas, VA). The case was approved by the University of Michigan's Institutional Review Board following written informed consent from the patient.

\section{Literature review}

A review of the literature identified 64 reported cases of $S$. salivarius meningitis, which are detailed further in table 1. The review was performed for indexed English- and nonEnglish-language articles using MEDLINE (National Library of Medicine, Bethesda, MD) for the search terms "meningitis" and "Streptococcus salivarius". Additional references were identified within bibliographies provided by MEDLINE-cited studies. Literature was reviewed through April 2011.

\section{Literature analysis}

Case reports were included in this analysis if they documented evidence of $S$. salivarius meningitis. Cases were assessed to characterize demographic features, signs and symptoms of infection, potential sources of infection, method of diagnosis, antimicrobial treatment regimens, and outcomes. Not all data were available from each report. Results were expressed as the total number of cases from which information was available among a total of 65 identified reports of $S$. salivarius meningitis (including the case presented here).

\section{Polymerase chain reaction}

The identity of $S$. salivarius from our patient was confirmed by sequencing the $16 \mathrm{~S}$ rRNA gene. Briefly, conserved primers were used to amplify the $16 \mathrm{~S}$ rRNA gene with high-fidelity taq polymerase (AmpliTaq Gold, Applied Biosystems, Inc). ${ }^{8}$ Amplicons were purified (QIAquick PCR Purification Kit, Qiagen, Inc) and sequenced at $2 \mathrm{X}$ coverage (Forward and Reverse directions) using standard Sanger-style sequencing (ABI 3730XL). Raw sequences were trimmed and aligned to a reference sequence from a $S$. salivarius strain (ATCC7073) as well as 20 additional reference $16 \mathrm{~S}$ rRNA sequences from various Streptococcus species. No differences were found between sequences from the strain in question and the $S$. salivarius reference strain.

\section{BinaxNOW ICT}

BinaxNOW ICT assays to detect $S$. pneumoniae antigen were conducted according to the manufacturer's instructions using the patient's urine. This test was also performed using CSF directly or broth cultures of the patient's S. salivarius CSF isolate or the ATCC reference strain 13419. The broth culture ICT tests were performed by culturing single colonies overnight in brain-heart infusion broth, wetting the ICT test strip with broth and then following the manufacturer's instructions.

\section{Case Report}

A 49-year-old obese woman with diabetes mellitus and hemodialysis-dependent end-stage renal disease presented with acute abdominal pain, nausea, vomiting, diarrhea, and headache. The morning of presentation, she developed a mild headache that progressively worsened throughout the day, along with neck stiffness. The patient noted low grade fevers, clear rhinorrhea, and a nonproductive cough for two weeks prior to presentation. She also complained of mild right ear pain for two to three days prior to hospitalization. There was no recent history of dental procedures, oral surgery, or spinal anesthesia.

The patient's medical history included hypertension, diabetic nephropathy, obesity, hyperthyroidism, and gout. Medications included calcium acetate, cinacalcet, glipizide, lovastatin, allopurinol, and methimazole. On initial examination the patient was in moderate distress from neck and head pain. She was afebrile; blood pressure was $143 / 70 \mathrm{mmHg}$, heart rate 105 beats/min, respiratory rate 18 breaths $/ \mathrm{min}$, and the room air, transcutaneous, arterial oxygen saturation was $99 \%$. The right tympanic membrane was dull with a small purulent airfluid level. She had clear rhinorrhea and meningismus with neck flexion. There were decreased breath sounds in the lung bases bilaterally, with no adventitious sounds. Cardiovascular examination was normal without murmurs. The abdomen was soft with active bowel sounds and diffuse tenderness to palpation. Her neurological examination was nonfocal with intact cranial nerves, normal reflexes, strength, and sensation to light touch. She was alert and oriented. No rash was present.

Laboratory testing revealed an initial white blood cell count (WBC) of $24.4 \times 10^{3} / \mathrm{uL}$ (normal range, 4.0-10.0 × $10^{3} / \mathrm{uL}$ ) with a differential of $93.6 \%$ neutrophils $(36.0 \%-75.0 \%)$, normal hemoglobin of $12.2 \mathrm{~g} / \mathrm{dL}(12.0-16 \mathrm{~g} / \mathrm{dL})$ and a platelet count of $225 \times 10^{3} / \mathrm{uL}\left(150-450 \times 10^{3} / \mathrm{uL}\right)$. An initial chest radiograph revealed low lung volumes with hypoventilatory changes and a right middle lobe and retrocardiac opacities consistent with either atelectasis or pneumonia. Concern for pneumonia resulted in empiric treatment with a single intravenous (IV) dose of levofloxacin.

After hospital admission the patient developed worsening headache, neck stiffness and pain exacerbated by neck flexion, an altered mental status with a decreased level of 


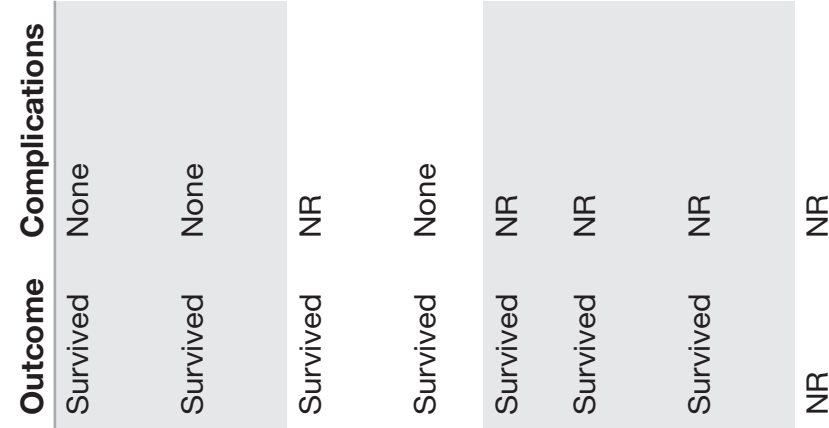

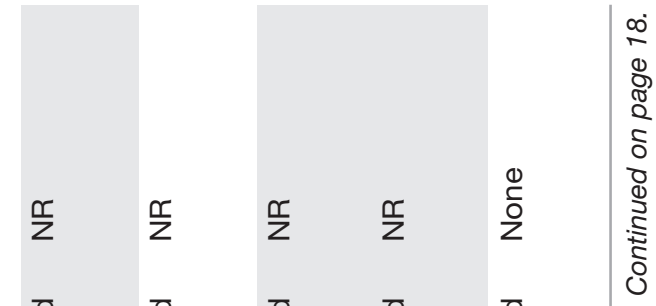

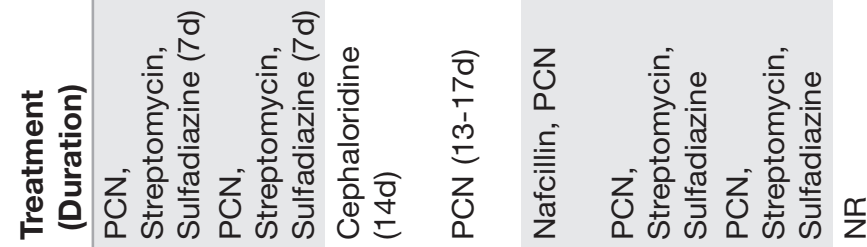

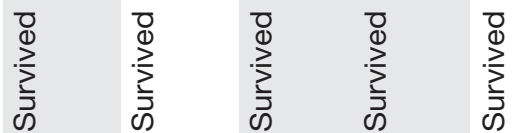

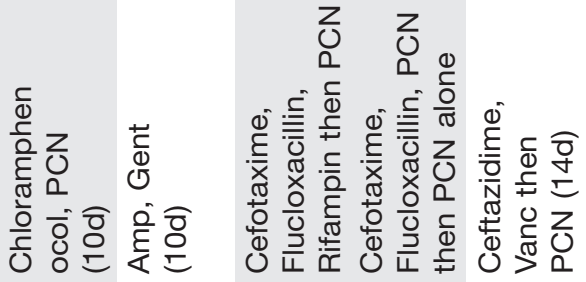

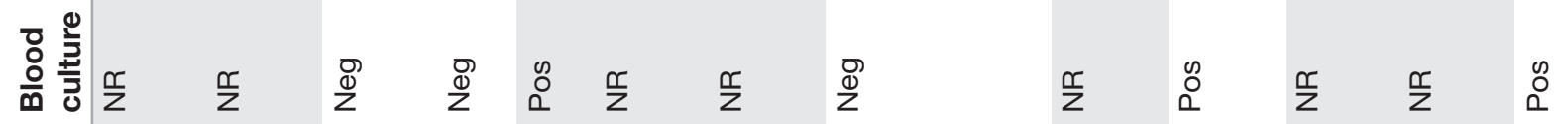

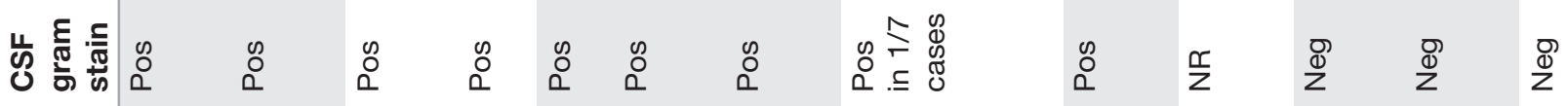

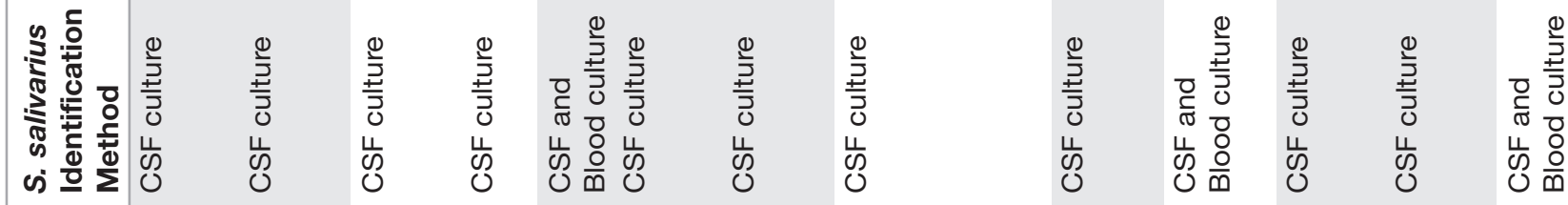

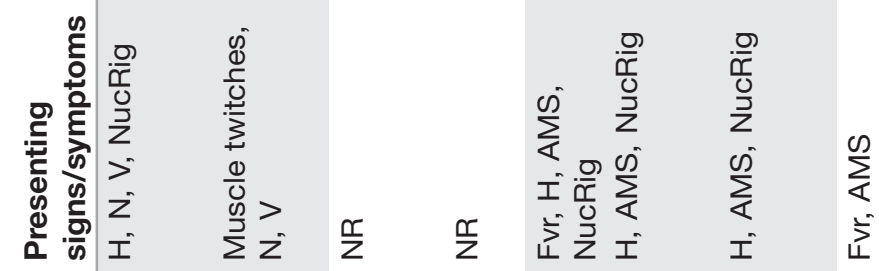

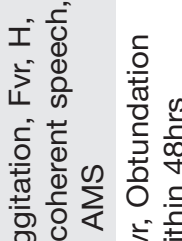
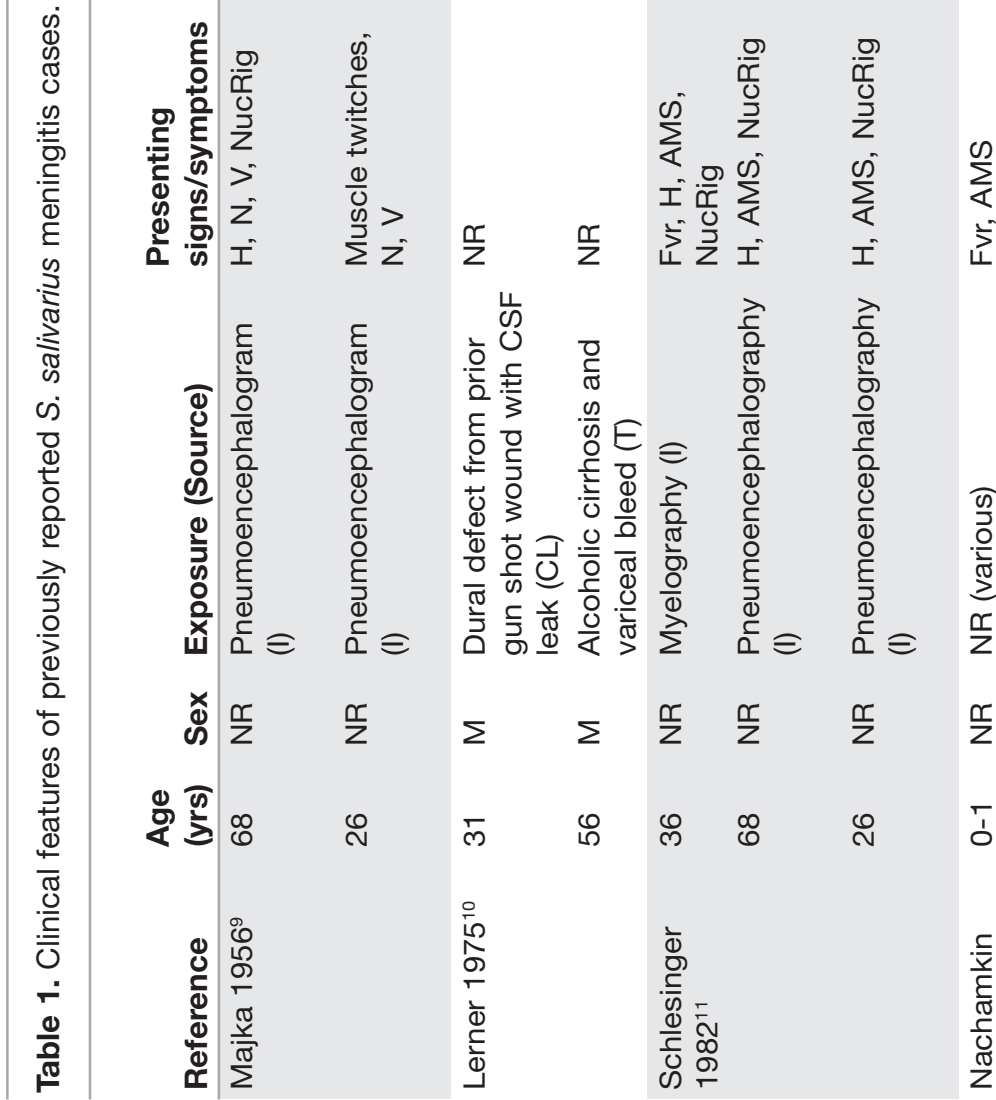

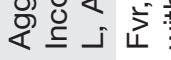

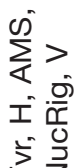

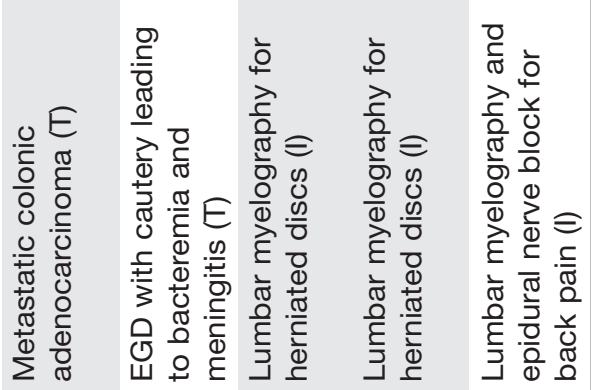

œ̊

ᄂ $\Sigma \quad \Sigma \quad$ ᄂ $\Sigma$

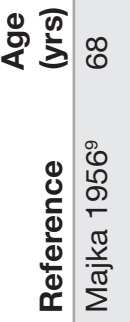

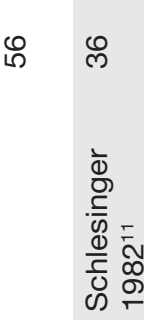

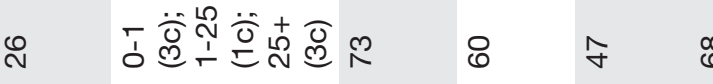

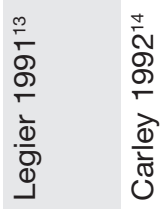

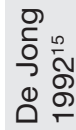

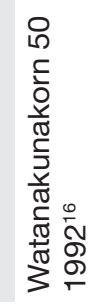




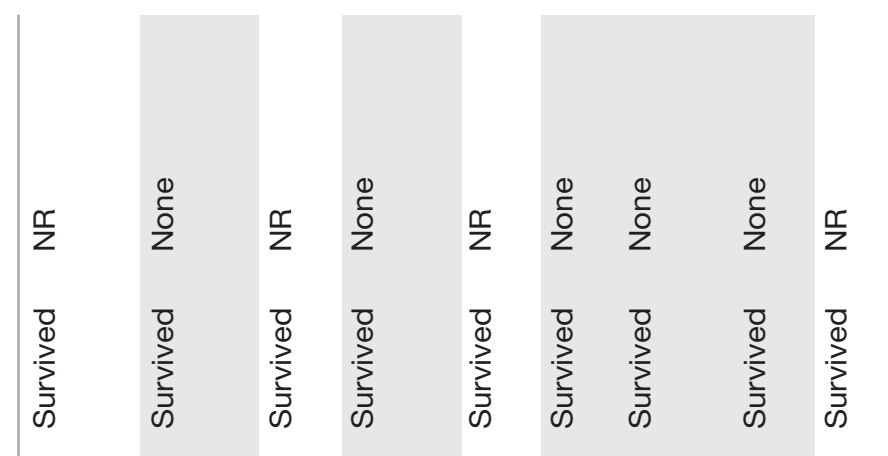

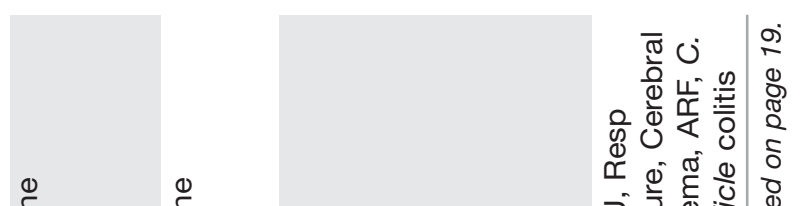

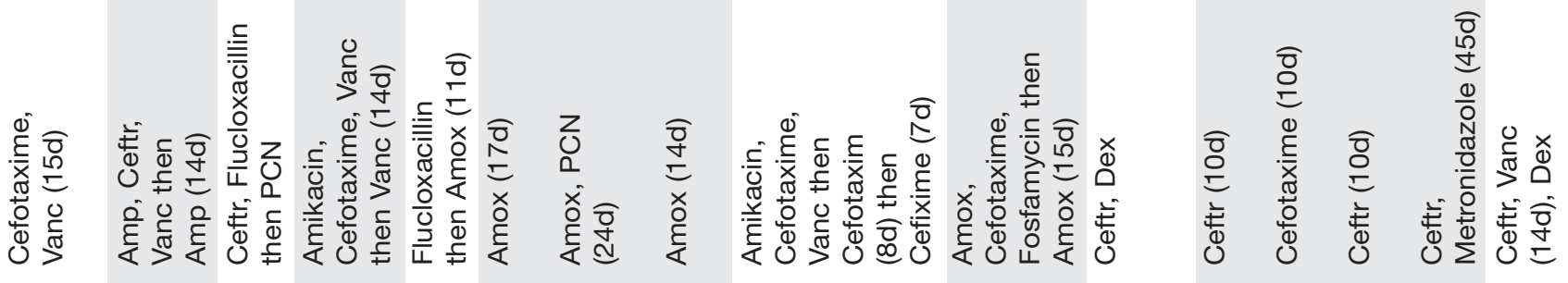

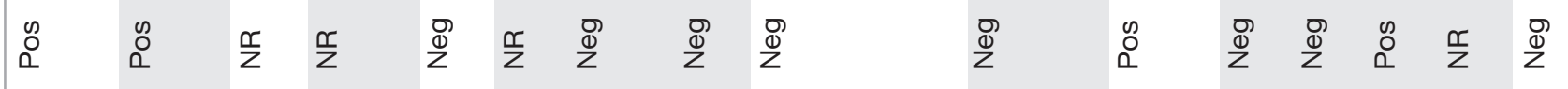

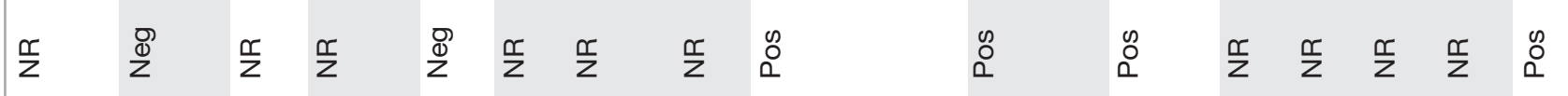

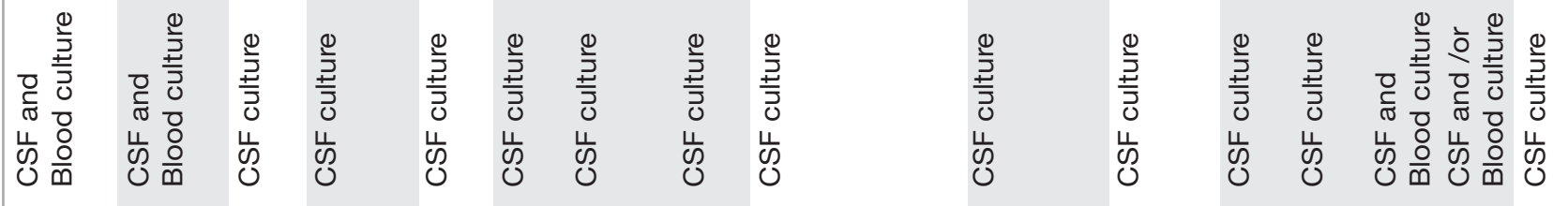

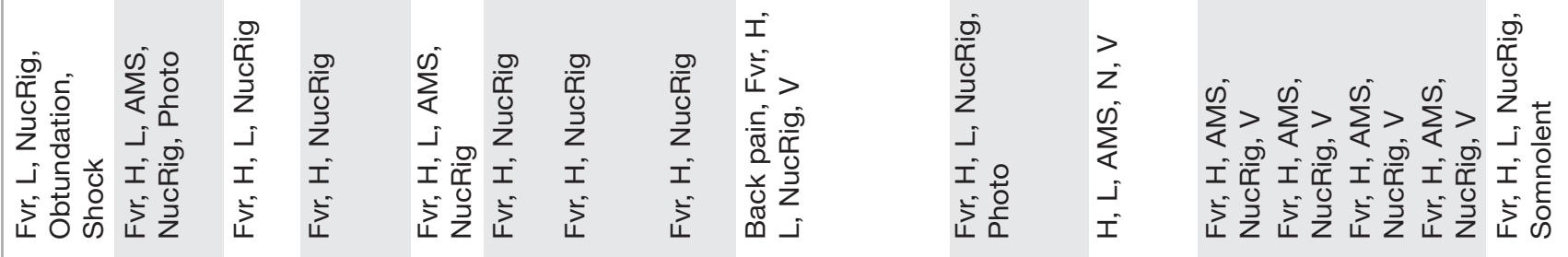

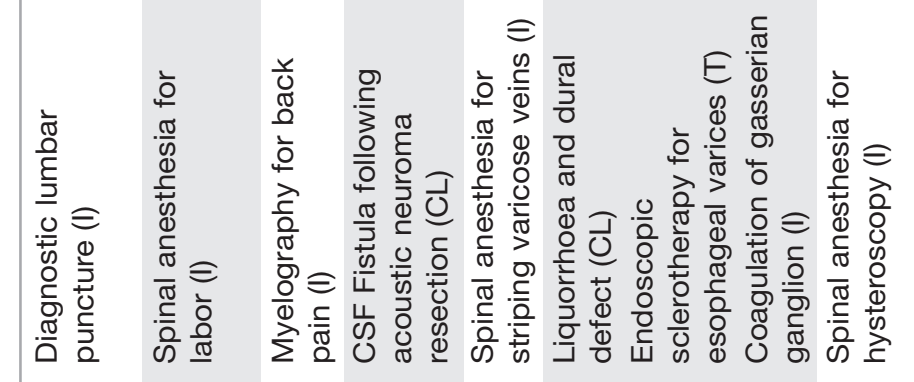

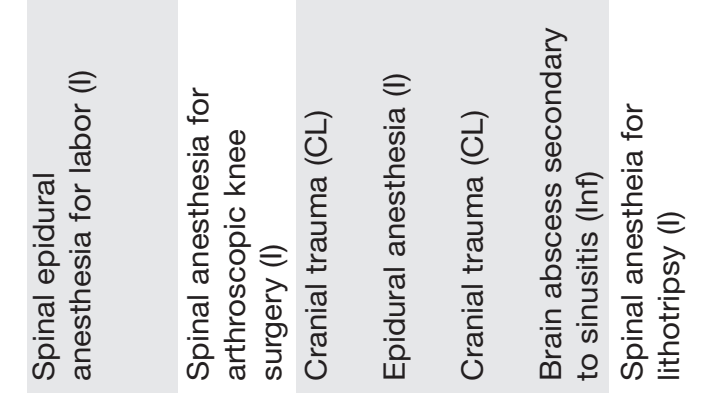
レ

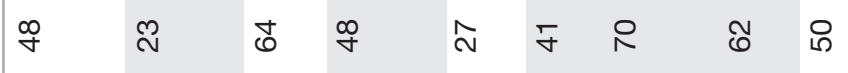
$\leftarrow \quad \Sigma \quad\llcorner\Sigma \Sigma \Sigma$

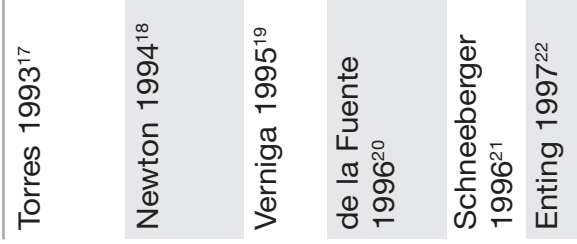
m

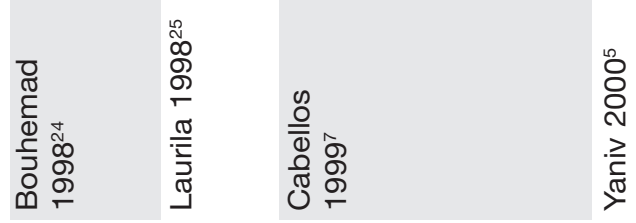




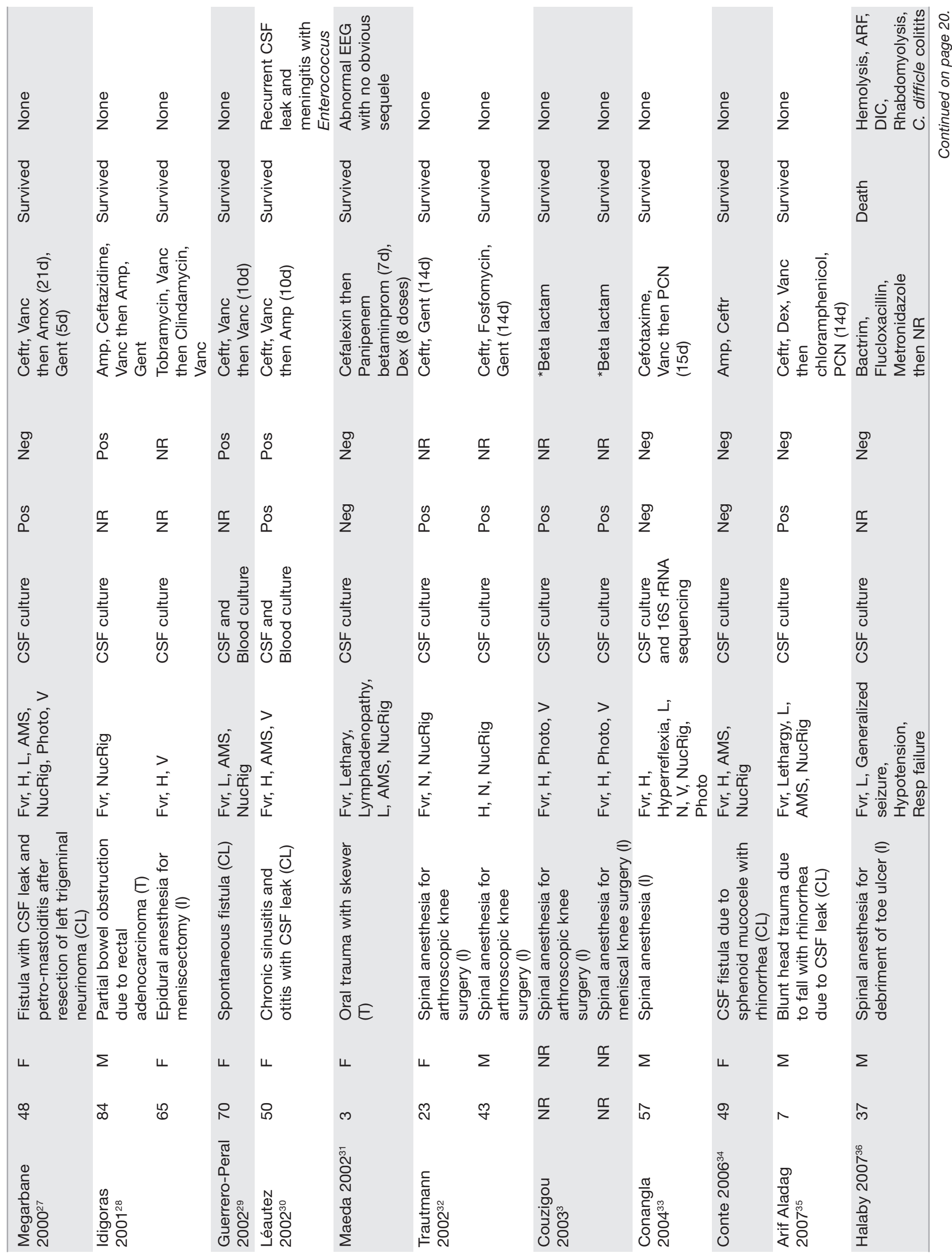




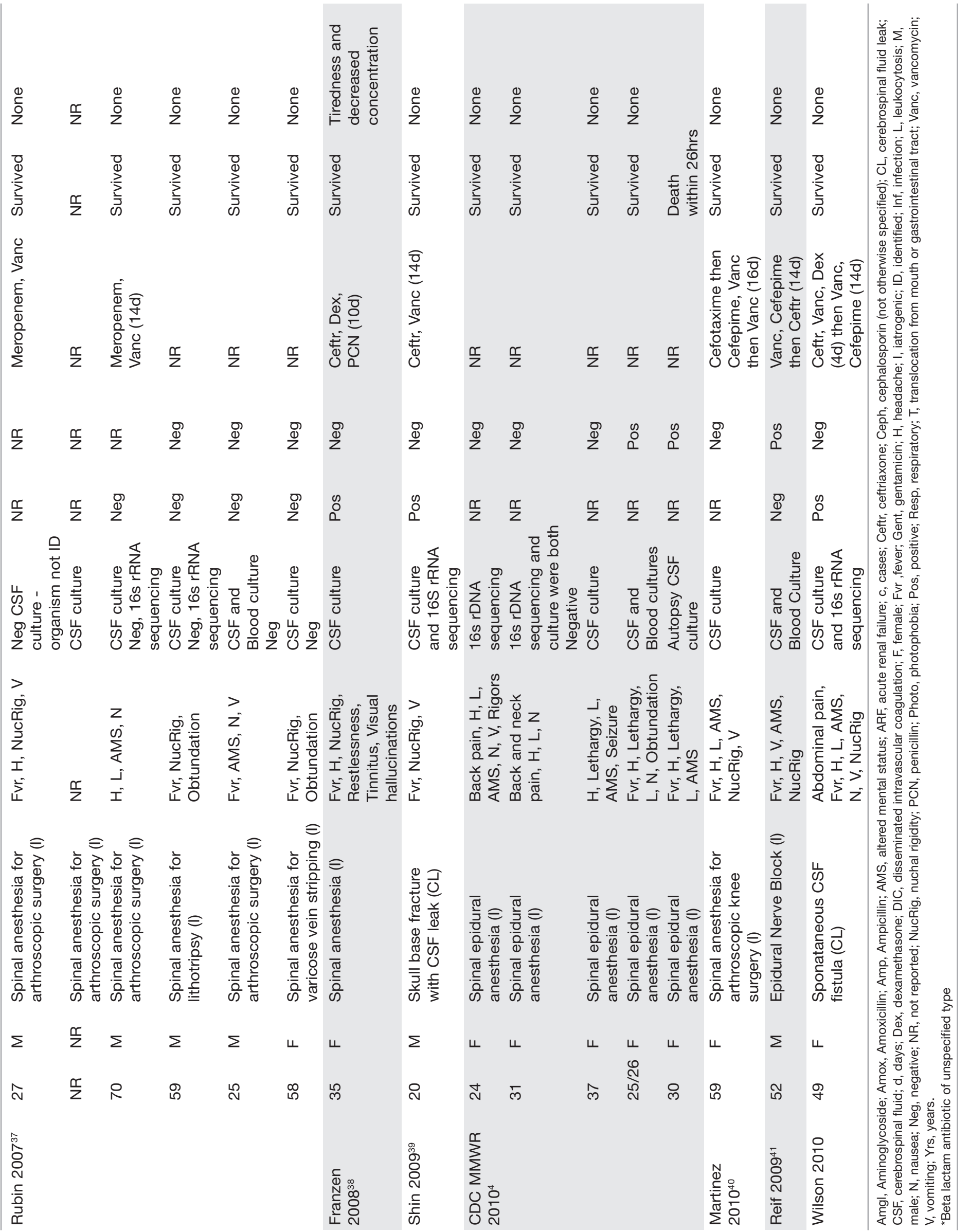



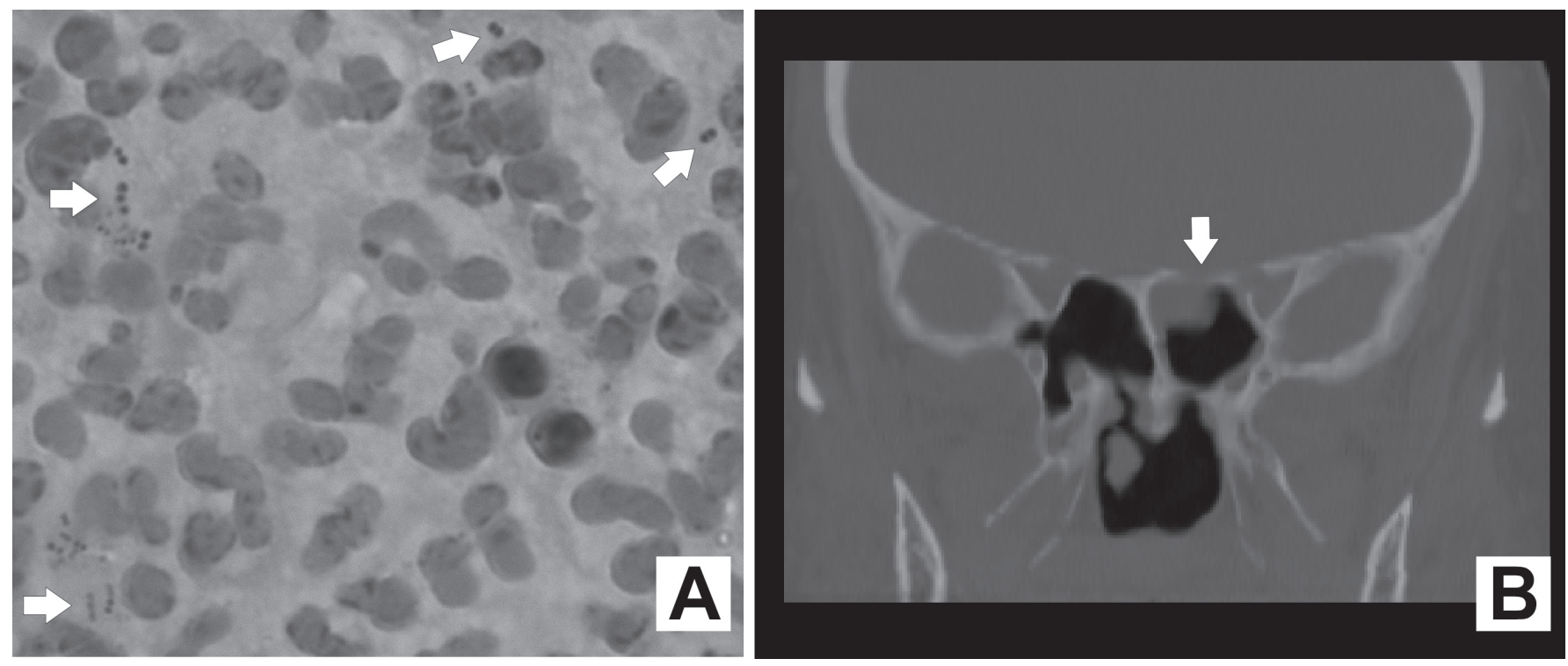

Figure 1. (A) Gram stain of cerebrospinal fluid demonstrated numerous polymorphonuclear leukocytes and Gram positive cocci in pairs and chains (arrows). (B) Coronal computed tomography scan image revealed a defect in the left planum sphenoidale with associated polypoid mucosal thickening in the sphenoid sinus (arrow).

consciousness, and emesis. Nuchal rigidity and pain with neck flexion were noted on exam (though Kernig and Brudzinski signs were absent). The patient's neurological status declined, with loss of orientation, recall, and concentration. The remainder of her neurological examination was unchanged. A non-contrasted computed tomography (CT) scan of the head demonstrated patchy opacification of the left mastoid air cells and a near complete opacification of the left sphenoid sinus. A lumbar puncture was performed approximately four hours after empiric antibiotics. The CSF was cloudy with $16,800 \mathrm{WBCs} / \mathrm{mm}^{3}$ (87\% polymorphonuclear leukocytes, $2 \%$ lymphocytes, and $11 \%$ histiocytes). The CSF glucose was $83 \mathrm{mg} / \mathrm{dL}(50-70 \mathrm{mg} / \mathrm{dL})$, while circulating blood glucose was $467 \mathrm{mg} / \mathrm{dL}(73-110 \mathrm{mg} / \mathrm{dL})$. The CSF protein was $322 \mathrm{mg} / \mathrm{dL}(15-45 \mathrm{mg} / \mathrm{dL})$. Gram stain of the CSF revealed gram positive cocci in pairs and short chains (figure 1A). Testing for cryptococcal antigen and enteroviruses, herpes simplex viruses 1 and 2, and adenoviruses by polymerase chain reaction (PCR) were negative. The CSF was screened for $S$. pneumoniae using a commerciallyavailable ICT, the BinaxNOW Urinary Antigen Test (Binax, Portland, ME), and was negative. Culture of the CSF yielded gram-positive cocci in pairs and chains later identified as $S$. salivarius. The identity was confirmed by sequencing the $16 \mathrm{~S}$ rRNA gene.

Unexpectedly, urine obtained from the patient was positive for $S$. pneumoniae antigen using the BinaxNOW ICT. In light of this positive urine test for $S$. pneumoniae, the patient's $S$. salivarius isolate was assayed in vitro for cross-reactivity with the BinaxNOW ICT, as noted in the methods section. This test was negative, as was a test using the ATCC laboratory reference strain of S. salivarius, ATCC 13419. There was low clinical suspicion that the patient had a concomitant $S$. pneumoniae infection because she lacked clinical symptoms of pneumonia, and her obesity was thought to be a source of hypoventalitory changes both on physical examination and chest radiograph.

Initial empiric therapy included vancomycin (1 g IV after dialysis), ceftriaxone (2 g IV every 12 hours) and dexamethasone (15 mg IV every 6 hours for 4 days). Within 12 hours of starting therapy the patient's mental status returned to baseline, and over the next 48 hours her headache and neck stiffness resolved. After the culture results returned, she was treated with cefepime $2 \mathrm{~g}$ IV and vancomycin $1 \mathrm{~g}$ IV (both after dialysis, three times per week) for 14 days. Broad spectrum antibiotics were maintained pending identification of the streptococcal species. There was complete neurological improvement, and no relapse since therapy was discontinued.

Following discharge, the patient had persistent intermittent occipital headaches and left-sided clear rhinorrhea. Drainage was exacerbated with leaning forward. She was evaluated with a non-contrast maxillofacial CT scan, which showed continued opacification of the left sphenoid sinus and left mastoid. Clinically, the left-sided rhinorrhea was suggestive of a CSF leak, and the drainage tested positive for $\beta 2$ transferrin by immunofixation. The patient also had a leftsided middle ear effusion that appeared to be consistent with retrograde filling of the middle ear space with CSF via the eustachian tube. A Stealth protocol thin cut maxillofacial CT was obtained, and on close inspection, a defect in the left planum sphenoidale was found. No tegmen tympani defects were noted (figure 1B). She underwent operative endoscopic evaluation, and two defects were found. A defect in the planum sphenoidale measured approximately $3 \mathrm{~mm}$ and was 
Table 2: Major Streptococcus Viridans Groups
S. mutans
S. salivarius
S. anginosus
S. mitis
S. sanguinus

repaired using a free mucosal graft. Another large linear defect was noted in the left cribiform overlying the olfactory tube, measuring about $1 \mathrm{~cm}$ by $3 \mathrm{~mm}$. This was adjacent to the septum, in a location that is classically difficult to repair. This was repaired with a vascularized pedicled nasal septal flap (Hadad-Bassagasteguy flap). Since the operation, there has been no further CSF leakage or other neurologic symptoms. Endoscopic evaluation shows complete coverage of the defects. The middle ear effusion persisted and was treated with myringotomy, although fluid was not cultured at the time of tube placement. The middle ear effusion has not recurred.

\section{Results of Literature Review}

Demographics/Predisposing Conditions

In addition to the case presented here, 64 published cases of $S$. salivarius meningitis were identified in the peer-reviewed

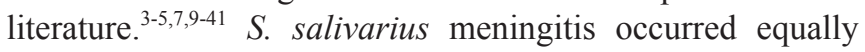
among men and women, with approximately $50 \%$ of cases in each gender ( 50 total cases reporting data). The age distribution of cases was broad, with the most common age deciles being 20 to 29 years of age and 50 to 59 years of age (figure 2). There was no clear evidence of predisposing co-morbid conditions in patients with meningitis, although in most case reports limited information was given on the medical history of each patient.

\section{Exposure/Inoculation}

The majority of cases of $S$. salivarius meningitis (39 of 58 cases, 67\%) were associated with iatrogenic causes, usually following epidural anesthesia or spinal myelography (table 1). In addition to the case reported here, there were 11 cases related to a leak of CSF. Of these leak-related cases, five developed following head trauma, ${ }^{7,10,35,39}$ two cases were complications from a neurosurgical procedure, ${ }^{20,27}$ two were due to spontaneous dural defects, ${ }^{22,29}$ one was due to a sphenoid mucocele, ${ }^{34}$ and a single case was associated with chronic sinusitis and otitis media (like our patient). ${ }^{30}$ The remaining cases (5 of 57) were associated with possible translocation from the gastrointestinal tract. ${ }^{10,13,14,22,28}$ There was a single case report of translocation from the mouth from trauma, ${ }^{31}$ and one report associated with a sinus infection. ${ }^{7}$

\section{Signs and Symptoms}

Streptococcus salivarius meningitis typically presented with classic findings of bacterial meningitis. The most commonly reported signs and symptoms were fever in 44 patients, headache in 40 patients, nuchal rigidity in 38 patients, altered mental status in 29 patients, nausea and vomiting in 27 patients (table 1). Leukocytosis was reported in 23 patients who had a median WBC count of $21.2 \times 10^{3} / \mathrm{uL}$ (with a range of $12.1-34.8 \times 10^{3} / \mathrm{uL}$ ) (table 1). Other less common presenting features included lethargy, obtundation, photophobia, seizures, sepsis, back pain, rigors, tinnitus, hyper-reflexia, and visual hallucinations.

\section{Duration of Symptoms}

A total of 50 reports of meningitis included data regarding the duration of symptoms prior to diagnosis. Of these, 45 cases $(90 \%)$ presented within one day of symptom onset. Thirtynine of the 50 cases $(78 \%)$ were iatrogenic. There was a nonsignificant trend for iatrogenic cases to get diagnosed sooner following the onset of symptoms than non-iatrogenic cases (22.3 \pm 2.7 hours vs. 39.6 hours to diagnosis) $(P=0.16$ by Student $t$-test).

\section{Microbiology and CSF Characteristics of Infection}

All reported cases were diagnosed on the basis of positive cultures or PCR from a lumbar puncture. Key biochemical and cellular characteristics of CSF obtained from case patients are represented in figure 3. The CSF glucose was typically low, with a mean of $28.1 \mathrm{mg} / \mathrm{dL}$, median of $22 \mathrm{mg} /$ $\mathrm{dL}$, and a range of $1-83 \mathrm{mg} / \mathrm{dL}$. Notably, five case reports indicated glucose levels $<10 \mathrm{mg} / \mathrm{dL}$, although specific values were not provided. ${ }^{4,18,28,38}$ The CSF protein was typically elevated with a mean of $499.2 \mathrm{mg} / \mathrm{dL}$, median of $434 \mathrm{mg} / \mathrm{dL}$, and a range of $34-1270 \mathrm{mg} / \mathrm{dL}$, with nine case reports indicating protein levels $>70 \mathrm{mg} / \mathrm{dL} \cdot{ }^{10,12,18,22,30,40,41}$ The median CSF WBC count was 5200 cells $/ \mathrm{mm}^{3}$ (mean of 7509 cells/ $\mathrm{mm}^{3}$, range $40-29,500$ cells $/ \mathrm{mm}^{3}$ ). This was always a neutrophil-predominant CSF, as the percentage of neutrophils ranged from $80 \%$ to $99 \%$ (median $95 \%$ ). The CSF gram stain was positive in 23 of 36 cases where results were reported. Blood cultures were positive in 13 of 40 cases (32.5\%) where data were available; however, data were not presented in these reports regarding the presence or absence of endocarditis.

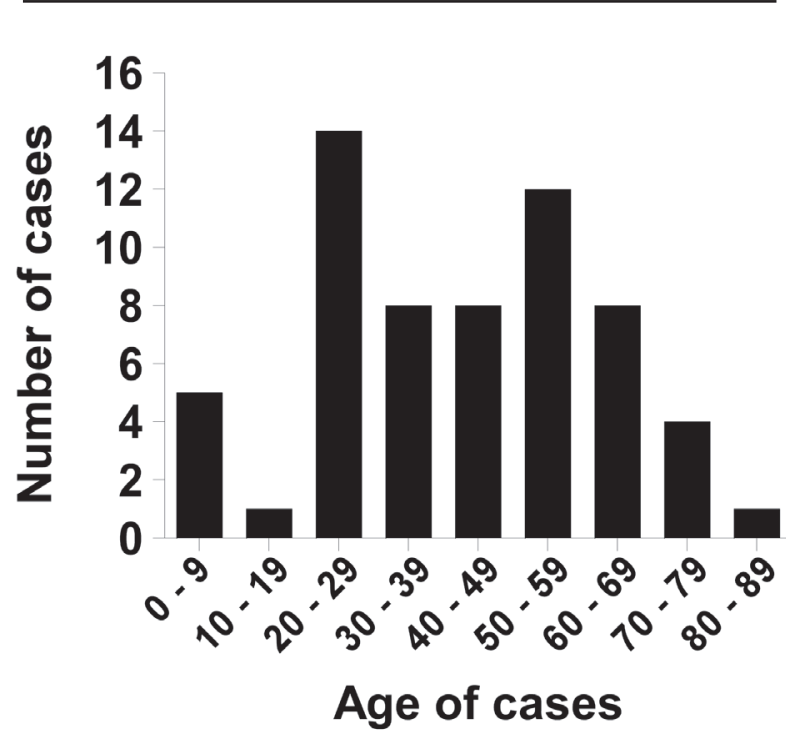

Figure 2. Age distribution of reported patients with S. salivarius meningitis ( $\mathrm{n}=60$ cases). 

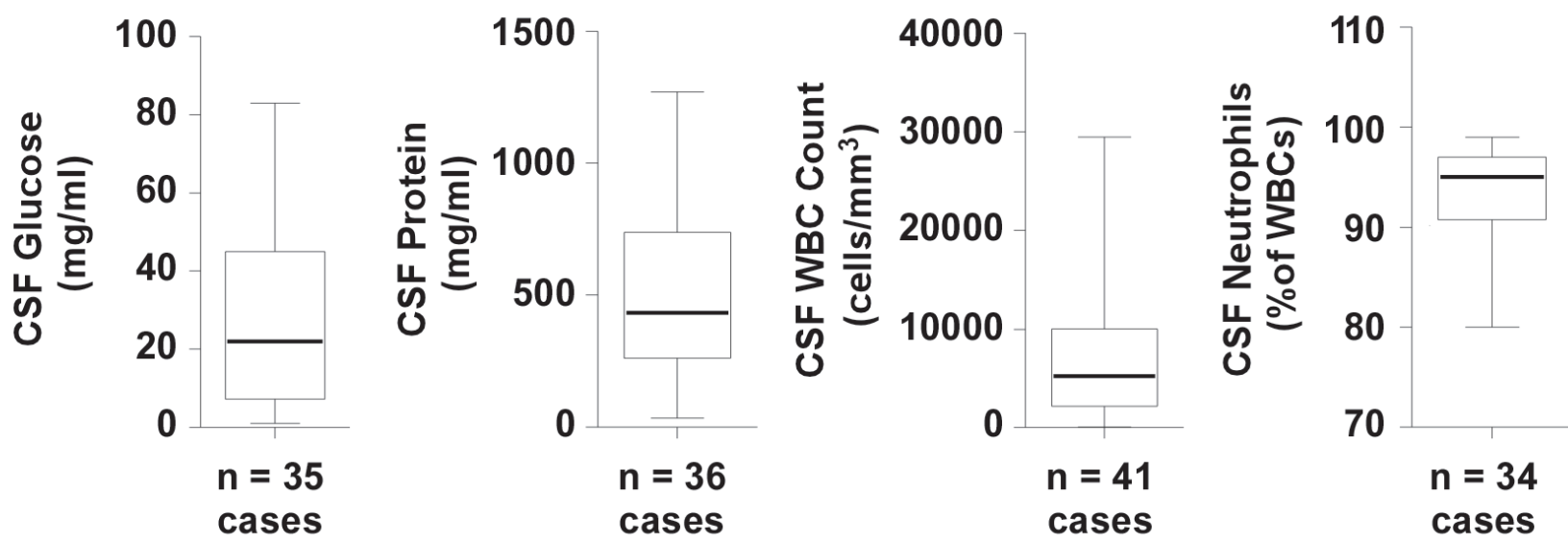

Figure 3. Cellular and biochemical features of cerebrospinal fluid obtained from patients with $S$. salivarius meningitis. Box and whisker plots demonstrate the median values (thick band near the middle of each box), the 25th and 75th percentile values (bottom and top of each box), and the range of reported values (the ends of the whiskers). WBC, white blood cells.

The majority of cases were determined to be S. salivarius based on positive CSF cultures in 62 of 65 cases $(95.3 \%)$. Only three cases had negative CSF cultures, and the bacterial pathogen was identified using alternative methods such as PCR of the $16 \mathrm{~S}$ rRNA gene., ${ }^{4,37}$

\section{Treatments}

The reported treatments of $S$. salivarius meningitis typically included a beta lactam antibiotic, most often either a penicillin or cephalosporin. The next most common medication was vancomycin. Dexamethasone was given in five cases, as well as in the present case described herein. ${ }^{5,25,31,35,38}$ The average duration of treatment was 14 days; although, duration was only reported in 33 of the 65 cases $(50 \%)$.

\section{Outcome and Follow-up}

In most cases of $S$. salivarius meningitis, patients survived without major sequelae of the infection, with 55 of 57 patients surviving and only 2 deaths (of those reporting outcome). ${ }^{4,36}$ Complications occurred in five patients, one of whom died. These complications included fatigue and decreased concentration in a 35-year-old female who sustained $S$. salivarius meningitis from epidural anesthesia. ${ }^{38}$ One patient with a petrous apex cholesteatoma developed a CSF leak and $S$. salivarius meningitis and then developed a recurrence of the leak leading to enterococcal meningitis. ${ }^{30}$ A 3-year-old girl who developed $S$. salivarius meningitis after oral penetrating trauma from a wooden skewer had an abnormal electroencephalogram but had no clinical sequelae. ${ }^{31}$ One patient required admission to the intensive care unit due to development of cerebral edema, respiratory failure, and acute renal failure. The patient survived the infection, and there was no mention of further sequel after discharge. ${ }^{5}$

\section{Discussion}

$S$. salivarius is a normal inhabitant of the human oral microbiome, ${ }^{42,43}$ and it is an uncommon human pathogen. ${ }^{44}$ In fact, recent attention has been placed on using this organism as a probiotic because of its ability to prevent colonization and proliferation of pathogenic streptococci and its capacity to suppress inflammatory responses from underlying host cells. ${ }^{43}$ However, as the present case and review of the literature suggest, this bacterium can cause life-threatening infections of the central nervous system. These are usually a complication of neurosurgical or anesthesia procedures, but can occur as a non-iatrogenic infection. To our knowledge, this is the largest review of previously published cases of $S$. salivarius meningitis to date.

Meningitis cases caused by viridans streptococci such as $S$. salivarius (table 2 ) have been increasingly reported since the mid- $20^{\text {th }}$ century. ${ }^{45}$ The increase in invasive procedures including neurosurgical procedures, spinal anesthesia, and prosthetic devices may account for the greater number of events. Given the probability of continued growth in the total number of invasive central nervous system procedures performed, this problem is unlikely to abate.

Our literature review identified 65 cases, including the present report, of bacterial meningitis secondary to $S$. salivarius. This review associated $S$. salivarius meningitis with CSF leaks (eg, head trauma, neurosurgical procedures, spontaneous leak) in $21 \%$ (12 of 58 cases reporting on the occurrence of leaks). Probable gastrointestinal translocation (including the mouth) occurred in 10\% (6 of 58) of patients for whom a source was identified, and there were iatrogenic causes in $67 \%$ (39 of 58) of cases. A source was not identified in only seven cases. The most common interventions associated with infection in the iatrogenic group were epidural anesthesia, spinal anesthesia, and myelography. S. salivarius is a commensal member of the oral mucosa, and many cases have been tracked to the oral microbiome of a healthcare provider performing a procedure on the case patient. ${ }^{19,32,36}$ This suggests that droplet transmission or contamination of sterile equipment by the operator is an important mechanism for inoculation. Thus, strict practices of aseptic technique 
during these procedures and use of a facemask in accordance with guidelines as published by the Healthcare Infection Control Practices Advisory Committee and American Society of Regional Anesthesia and Pain Medicine will be important to limiting the incidence of these events. ${ }^{4,46}$

Microbiological diagnoses were established in most patients by CSF culture, blood culture, or CSF PCR when no pathogen was identified. In the case presented here, the CSF culture was used to identify $S$. salivarius, and PCR with gene sequencing analysis was used to confirm its identity. Interestingly, our patient's urine pneumococcal antigen ICT test was positive. However, neither a pure culture of her CSF isolate nor a reference ATCC strain of $S$. salivarius crossreacted in vitro with this test. While cross-reactions have been noted with the structurally-similar $S$. oralis and $S$. mitis,${ }^{47,48} S$. salivarius should not be added to the list of organisms that cause a false-positive ICT for pneumococcus. It is unlikely that the false positive ICT result adversely affected our patient's care, as her isolate of $S$. salivarius was relatively antibiotic-susceptible (MIC to ceftriaxone $=0.5 \mu \mathrm{g} / \mathrm{ml}$ ).

To the best of our knowledge, only two cases of antibiotic resistant $S$. salivarius meningitis have been reported to date. ${ }^{5,40}$ However according to a study by van Doern et al ${ }^{49}$ $20 \%$ of S. salivarius were resistant to ceftriaxone and $17 \%$ had high resistance to penicillin. These rates of resistance should be of concern to clinicians, leading to aggressive attempts to establish a microbiological diagnosis that allows for appropriately "directed" therapy.

Although the present review was intended to provide a comprehensive overview of the state of knowledge about $S$. salivarius meningitis, it has several limitations. For example, because many of the cases are single reports, reporting bias may affect our findings. In addition, there was not a complete set of data for each case. Although we identified the most common presenting symptoms, causes, and treatment algorithms for many cases, some important data were not reported, including descriptions of the physical examination or rationales to justify antibiotic choices. Most data suggest that $S$. salivarius meningitis presents with classic findings of bacterial meningitis.

In summary, S. salivarius is an important cause of iatrogenic meningitis. Strict use of aseptic techniques including a facemask during diagnostic and surgical procedures may limit the incidence of this infectious complication. Thorough investigation of potential cases should be performed in order to prevent potential outbreaks from a single source. When a patient develops $S$. salivarius meningitis without a predisposing iatrogenic or traumatic event, a search for a CSF leak may be helpful. Most patients who develop infection with $S$. salivarius meningitis can safely be treated with a penicillin, third generation cephalosporin, or vancomycin with a good clinical outcome and cure.

\section{References}

1. Mace SE. Acute bacterial meningitis. Emerg Med Clin North Am 2008;26:281-317, viii.

2. Lynch JP, 3rd, Zhanel GG. Streptococcus pneumoniae: epidemiology and risk factors, evolution of antimicrobial resistance, and impact of vaccines. Curr Opin Pulm Med 2010;16:217-225.

3. Couzigou C, Vuong TK, Botherel AH, Aggoune M, Astagneau P. Iatrogenic Streptococcus salivarius meningitis after spinal anaesthesia: need for strict application of standard precautions. J Hosp Infect 2003;53:313-314.

4. Centers for Disease Control and Prevention (CDC). Bacterial meningitis after intrapartum spinal anesthesia - New York and Ohio, 2008-2009. MMWR Morb Mortal Wkly Rep 2010;59:65-69.

5. Yaniv LG, Potasman I. Iatrogenic meningitis: an increasing role for resistant viridans streptococci? Case report and review of the last 20 years. Scand J Infect Dis 2000;32:693-696.

6. Baer ET. Post-dural puncture bacterial meningitis. Anesthesiology 2006;105:381-393.

7. Cabellos C, Viladrich PF, Corredoira J, Verdaguer R, Ariza J, Gudiol F. Streptococcal meningitis in adult patients: current epidemiology and clinical spectrum. Clin Infect Dis 1999;28:1104-1108.

8. Antonopoulos DA, Huse SM, Morrison HG, Schmidt TM, Sogin ML, Young VB. Reproducible community dynamics of the gastrointestinal microbiota following antibiotic perturbation. Infect Immun 2009;77:2367-2375.

9. Majka FA, Gysin WM, Zaayer RL. Streptococcus salivarius meningitis following diagnostic lumbar puncture. Nebr State Med J 1956;41:279-281.

10. Lerner PI. Meningitis caused by Streptococcus in adults. J Infect Dis 1975;131:S9-S16.

11. Schlesinger JJ, Salit IE, McCormack G. Streptococcal meningitis after myelography. Arch Neurol 1982;39:576-577.

12. Nachamkin I, Dalton HP. The clinical significance of streptococcal species isolated from cerebrospinal fluid. Am J Clin Pathol 1983;79:195-199.

13. Legier JF. Streptococcus salivarius meningitis and colonic carcinoma. South Med J 1991;84:1058-1059.

14. Carley NH. Streptococcus salivarius bacteremia and meningitis following upper gastrointestinal endoscopy and cauterization for gastric bleeding. Clin Infect Dis 1992;14:947-948.

15. de Jong J, Barrs AC. Lumbar myelography followed by meningitis. Infect Control Hosp Epidemiol 1992;13:74-75.

16. Watanakunakorn C, Stahl C. Streptococcus salivarius meningitis following myelography. Infect Control Hosp Epidemiol 1992;13:454.

17. Torres E, Alba D, Frank A, Diez-Tejedor E. Iatrogenic meningitis due to Streptococcus salivarius following a spinal tap. Clin Infect Dis 1993;17:525-526.

18. Newton JA, Jr., Lesnik IK, Kennedy CA. Streptococcus salivarius meningitis following spinal anesthesia. Clin Infect Dis 1994;18:840-841.

19. Veringa E, van Belkum A, Schellekens H. Iatrogenic meningitis by Streptococcus salivarius following lumbar puncture. J Hosp Infect 1995;29:316-318.

20. de la Fuente Aguado J, Moreno Sanjuan JA, Fernandez Villar A, Otero Varela I, Conde C. [Meningitis caused by Streptococcus salivarius]. [Article in Spanish] An Med Interna 1996;13:355.

21. Schneeberger PM, Janssen M, Voss A. Alpha-hemolytic streptococci: a major pathogen of iatrogenic meningitis following lumbar puncture. Case reports and a review of the literature. Infection 1996;24:29-33.

22. Enting RH, de Gans J, Blankevoort JP, Spanjaard L. Meningitis due to viridans streptococci in adults. J Neurol 1997;244:435-438. 
23. Kaiser E, Suppini A, de Jaureguiberry JP, Paris JF, Quinot JF. [Acute Streptococcus salivarius meningitis after spinal anesthesia]. [Article in French] Ann Fr Anesth Reanim 1997; 16:47-49.

24. Bouhemad B, Dounas M, Mercier FJ, Benhamou D. Bacterial meningitis following combined spinal-epidural analgesia for labour. Anaesthesia 1998;53:292-295.

25. Laurila JJ, Kostamovaara PA, Alahuhta S. Streptococcus salivarius meningitis after spinal anesthesia. Anesthesiology 1998;89:1579-1580.

26. Molinier S, Paris JF, Brisou P, Amah Y, Morand JJ, Alla P, Carli P. [2 cases of iatrogenic oral streptococcal infection: meningitis and spondylodiscitis]. [Article in French] Rev Med Interne 1998;19:568-570.

27. Megarbane B, Casetta A, Esvant H, Marchal P, Axler O, Brivet FG. Streptococcus salivarius acute meningitis with latent petromastoiditis. Scand J Infect Dis 2000;32:322-323.

28. Idigoras P, Valiente A, Iglesias L, Trieu-Cout P, Poyart C. Meningitis due to Streptococcus salivarius. J Clin Microbiol 2001;39:3017.

29. Guerrero-Peral AL, Guerrero-Peral AB. [Meningitis due to Streptococcus salivarius and spontaneous fistula: a case report]. [Article in Spanish] Rev Neurol 2002;35:799-800.

30. Leautez S, Bironneau E, Espaze E, Bordure P, Raffi F. [Streptococcus salivarius meningitis with bacteriaemia in a patient with petrous apex cholesteatoma.] [Article in French] Med Mal Infect 2002;32:49-51.

31. Maeda H, Shinoda G, Kuroki S, Tsutsui T, Kubota M, Haruta T. [Streptococcus salivarius meningitis after oral trauma by a skewer: a case report]. [Article in Japanese.] Kansenshogaku Zasshi 2002;76:72-75.

32. Trautmann M, Lepper PM, Schmitz FJ. Three cases of bacterial meningitis after spinal and epidural anesthesia. Eur J Clin Microbiol Infect Dis 2002;21:43-45.

33. Conangla G, Rodriguez L, Alonso-Tarres C, Avila A, de la Campa AG. [Streptococcus salivarius meningitis after spinal anesthesia]. [Article in Spanish] Neurologia 2004;19:331333.

34. Conte A, Chinello P, Civljak R, Bellussi A, Noto P, Petrosillo N. Streptococcus salivarius meningitis and sphenoid sinus mucocele. Case report and literature review. J Infect 2006;52:e27-e30.

35. Arif Aladag M, Refik M, Halil Ozerol I, Tarim O. Posttraumatic Streptococcus salivarius meningitis in a child. Pediatr Int 2007;49:112-114.

36. Halaby T, Leyssius A, Veneman T. Fatal bacterial meningitis after spinal anaesthesia. Scand J Infect Dis 2007;39:280-283.

37. Rubin L, Sprecher H, Kabaha A, Weber G, Teitler N, Rishpon $\mathrm{S}$. Meningitis following spinal anesthesia: 6 cases in 5 years. Infect Control Hosp Epidemiol 2007;28:1187-1190.

38. Franzen N, Bach LF. [Meningitis following spinal anaesthesia]. [Article in Danish] Ugeskr Laeger 2008;170:1941.

39. Shin KS, Shin DI, Shim WS, Rim BC, Bae IH, Lee SY, Ryu DH, Kim EJ, Son BR. A case of Streptococcus salivarius meningitis in a patient with cerebrospinal fluid rhinorrhea after skull base fracture. Korean J Clin Microbiol 2009; 12:92-96.

40. Martinez LJ, Robles M, Isach N, Ribell M. [Acute iatrogenic meningitis due to Streptococcus salivarius after spinal anesthesia]. [Article in Spanish] Rev Esp Anestesiol Reanim 2010;57:252-253.

41. Reif S, Roller J, Rawling R, Granato P. Iatrogenic Streptococcus Salivarius Meningitis. Clinical Microbiology Newsletter 2009;31:6-7

42. Safford CE, Sherman JM, Hodge HM. Streptococcus salivarius. J Bacteriol 1937;33:263-274
43. Guglielmetti S, Taverniti V, Minuzzo M, Arioli S, Stuknyte M, Karp M, Mora D. Oral bacteria as potential probiotics for the pharyngeal mucosa. Appl Environ Microbiol 2010;76:39483958.

44. Andrewes FW, Horder TJ. A study of the streptococci pathogenic for man. Lancet 1906;168:775-783.

45. Kyser FA. Streptococcus viridans meningitis with pneumonia. Ill Med J 1947;91:81-83.

46. Siegel JD, Rhinehart E, Jackson M, Chiarello L. 2007 Guideline for Isolation Precautions: Preventing Transmission of Infectious Agents in Health Care Settings. Am J Infect Control 2007;35:S65-S164.

47. Alonso-Tarrés C, Cortés-Lletget $\mathrm{C}$, Casanova $\mathrm{T}$, Domènech $\mathrm{A}$. False-positive pneumococcal antigen test in meningitis diagnosis. Lancet 2001;358:1273-1274.

48. Gillespie SH, McWhinney PH, Patel S, Raynes JG, McAdam KP, Whiley RA, Hardie JM. Species of alpha-hemolytic streptococci possessing a C-polysaccharide phosphorylcholine-containing antigen. Infect Immun 1993;61:3076-3077.

49. Doern GV, Ferraro MJ, Brueggemann AB, Ruoff KL. Emergence of high rates of antimicrobial resistance among viridans group streptococci in the United States. Antimicrob Agents Chemother 1996;40:891-894.

\section{Author Affiliations}

Megan Wilson, MD*; Ryan Martin, MD'; Seth T. Walk, PhD'; Carol Young, MT(ASCP); Sylvia Grossman, MT(ASCP)*;

Erin Lin McKean, MDs; David M. Aronoff, MDt, ,,\#

*Department of Internal Medicine, University of Michigan

Medical School, Ann Arbor, MI 48109

Division of Infectious Diseases, University of Michigan

Medical School, Ann Arbor, MI 48109

Clinical Microbiology Laboratories, Department of

Pathology, University of Michigan Medical School,

Ann Arbor, MI 48109

sDepartment of Otolaryngology, University of Michigan

Medical School, Ann Arbor, MI 48109

Graduate Program in Immunology and Program in

Biomedical Sciences, University of Michigan Medical

School, Ann Arbor, MI 48109

\#Department of Microbiology and Immunology, University of Michigan Medical School, Ann Arbor, MI 48109 